Planetary Systems in the Universe - Observation, Formation and Evolution

Proceedings IAU Symposium No. 202, (C)2004 IAU

Alan Penny, Pawel Artymowicz, Anne-Marie Lagrange, \& Sara Russell, eds.

\title{
CORALIE-ELODIE new planets and planetary systems. Looking for fossil traces of formation and evolution
}

\author{
S. Udry, M. Mayor and D. Queloz \\ Geneva Observatory, CH-1290 Sauverny, Switzerland
}

\begin{abstract}
HD $6434 \mathrm{~b}, \mathrm{HD} 19994 \mathrm{~b}$, HD 83443 c, HD 92788 b, HD 121504 b, HD 190228 b ) are announced as part of our planet-search programmes in the northern and southern hemispheres. HD $83443 \mathrm{c}$ is member of a 2-planet system with Saturnian and sub-Saturnian masses. Another system including a planet + a very low-mass brown dwarf orbiting HD 168443 is also presented. These 2 new systems and the new planetary detections rise to 25 the number of ELODIE and CORALIE candidates with minimum masses $\leq 20 \mathrm{M}_{\text {Jup }}$. The orbital element distributions of giant-planet candidates, like the secondary mass function, the eccentricity and period distributions, compared to the equivalent distributions for spectroscopic binaries, strongly suggest different formation mechanisms for the two populations.
\end{abstract}

\section{Introduction}

New enthusiastic results in the domain of extra-solar planets are popping up on an almost daily basis. From one meeting to the next, not only new candidates are announced by the teams of "planet hunters" monitoring high-precision radial velocities (RV) but also number of various approaches to the field are explored, trying to better understand planetary formation as new observational results often tend to set new questions rather than bring definitive answers to the variety of explanations proposed for the observed properties of these systems.

In this contribution new discoveries are presented and the global properties of the extra-solar planet sample as a whole are discussed as well. The first part of the paper will be dedicated to the description of new planet candidates obtained in the frame of two large planet-search programmes around solar-type stars, in which our group in Geneva is involved, namely the ELODIE high-precision RV survey in Haute-Provence (France) for the northern sky and the CORALIE planet-search programme at ESO-La Silla (Chile) in the south.

Among the new ELODIE results we will focus on the spectroscopic transit of the planet in front of HD 209458 and on the new planetary companion orbiting the star HD 190228. The CORALIE programme is rich in new prominent results with 5 new planets around HD 6434, HD 19994, HD 83443, HD 92788, and HD 121504 . HD 83443 turns out to be the $2^{\text {nd }}$ known exoplanetary system, hosting two very low-mass planets, respectively close to and of half the mass of Saturn. An additional $3^{\text {rd }}$ new system composed of a massive planet + a very low-mass brown dwarf $\left(15.1 \mathrm{M}_{\mathrm{Jup}} / \sin i\right)$ orbiting HD 168443 is presented as well.

Finally, the end part of this contribution will be devoted to a global description of the extra-solar planets properties in terms of orbital element distributions 
Table 1. Stellar and orbital parameters for $14 \mathrm{Her}$ and HD 168443

\begin{tabular}{|c|c|c|c|c|}
\hline & $14 \mathrm{Her} \mathrm{b}$ & HD $168443 \mathrm{~b}$ & HD $168443 \mathrm{c}$ & Units \\
\hline$S p$ & $\overline{K O V}$ & \multicolumn{2}{|c|}{ G5 } & \\
\hline$V$ & 6.6 & \multicolumn{2}{|c|}{6.9} & [mag] \\
\hline$M_{1}$ & 0.79 & \multicolumn{2}{|c|}{0.92} & {$\left[\mathrm{M}_{\odot}\right]$} \\
\hline$P$ & $1787.5 \pm 16.5$ & $58.117 \pm 0.014$ & $1667 \pm 48$ & days \\
\hline$T$ & $49579.3 \pm 23.3$ & $51558.36 \pm 0.12$ & $50269.5 \pm 36$ & [JD-2400000] \\
\hline$e$ & $0.34 \pm 0.02$ & $0.526 \pm 0.008$ & $0.265 \pm 0.049$ & \\
\hline$V$ & -13.842 & \multicolumn{2}{|c|}{-48.744} & \multirow{3}{*}{$\begin{array}{l}{\left[\mathrm{kms}^{-1}\right]} \\
{[\mathrm{deg}]} \\
{\left[\mathrm{ms}^{-1}\right]}\end{array}$} \\
\hline$\omega$ & $22.05 \pm 3.89$ & $172.2 \pm 1.1$ & $59 \pm 5$ & \\
\hline$K$ & $91 \pm 2$ & $473 \pm 6$ & $288 \pm 13$ & \\
\hline$m_{2}$ & 4.2 & 7.2 & 15.1 & {$\left[\mathrm{M}_{\mathrm{Jup}} / \sin i\right]$} \\
\hline$a$ & 2.68 & 0.29 & 2.67 & {$[\mathrm{AU}]$} \\
\hline$N$ & $84^{a}$ & \multicolumn{2}{|c|}{$58^{b}+30^{c}$} & \\
\hline RMS & 12.7 & \multicolumn{2}{|c|}{7.6} & {$\left[\mathrm{~ms}^{-1}\right]$} \\
\hline
\end{tabular}

${ }^{a}$ ELODIE, ${ }^{b}$ CORALIE, ${ }^{c}$ HIRES (from Marcy et al. 1999b)

emphasizing their differences from the equivalent distributions for stellar companions to solar-type stars.

\section{The ELODIE high-precision radial-velocity survey}

Our RV survey in the northern hemisphere to search for planets orbiting solartype stars is a Swiss-French collaboration including the Geneva, the Grenoble and the Haute-Provence observatories. The high-precision RVs are measured by numerically cross-correlating the ELODIE stellar spectra with a solar-type template (Baranne et al. 1996). ELODIE is a two-fiber fed high-resolution echelle spectrograph $(R \simeq 42000)$ mounted on the 193-cm telescope of the Haute-Provence Observatory. The two fibers are used to simultaneously take the stellar spectra and follow with a ThAr calibration lamp the instrumental drifts due to environmental effects (temperature and pressure changes). After the introduction in the fiber paths of double-scrambling devices, the achieved precision for RV measurements is now of $8-10 \mathrm{~ms}^{-1}$.

The ELODIE planet-search sample is magnitude-limited and consists of a $\sim 350 \mathrm{~F}-\mathrm{K}$ dwarfs of the solar vicinity. Its is an extension of the original MayorQueloz sample out of which $51 \mathrm{Peg}$ b, the first extra-solar planet around a solartype star, was discovered (Mayor \& Queloz 1995). In addition to this very mediatic early success of the programme, 4 additional planetary companions have been detected with ELODIE:

- Gl 876 b (Delfosse et al. 1999; also found by Marcy et al. 1999a) orbiting an M4 dwarf and discovered as part of a binarity study of M dwarfs.

- $14 \mathrm{Her}$ b; the long-period orbit is completely covered now. Updated orbital elements and RV curves are given in Table 1 and Fig. 1 (left).

- HD 209458 b; ELODIE and CORALIE measurements combined with HIRES data obtained at Keck 1 by D. Latham et al. allowed us to precisely determine the epoch of the planetary transit (Mazeh et al. 2000) then used by Charbonneau et al. (2000) to observed the photometric transit twice in September 1999. The transit was also independently observed by Henry et al. (2000). The pho- 

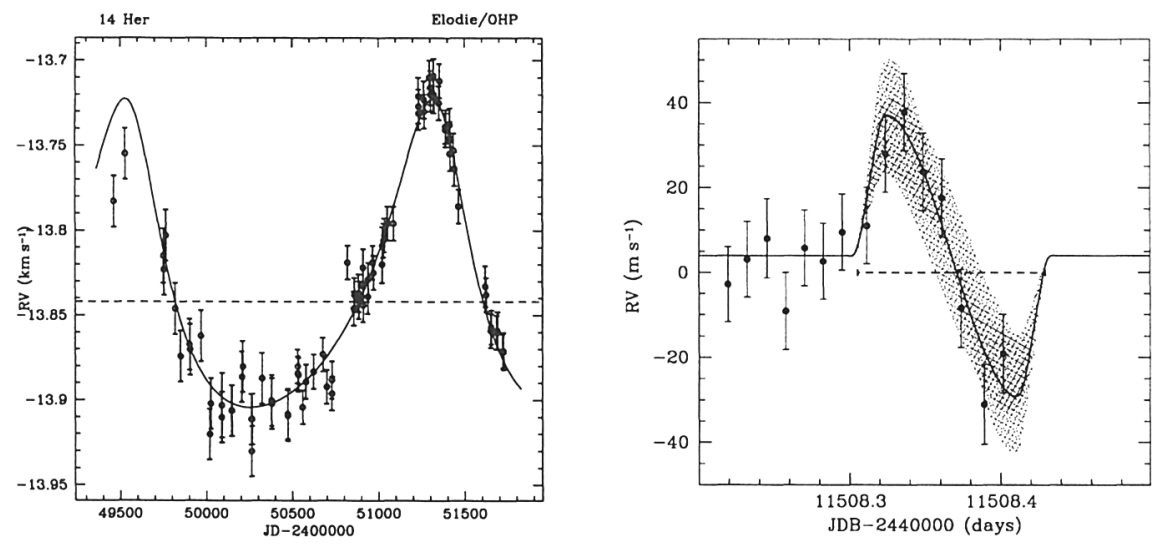

Figure 1. Left. RVs and updated Keplerian solution for $14 \mathrm{Her}$. Right. ELODIE spectroscopic transit of HD 209458: RVs and best solution for the anomaly curve corrected from the orbital motion (Queloz et al. 2000). The $1 \sigma$ confidence level is indicated by the hatched area

tometric transits brought the proof of the gaseous nature of HD 209458 (Mazeh et al. 2000). Further results on the geometry of the system are obtained by the spectroscopic observation of the transit with ELODIE (next section).

- The new massive planet $\left(m_{2} \sin i=5 \mathrm{M}_{\mathrm{Jup}}\right)$ orbiting HD 190228 on a long-period $(P=1161 \mathrm{~d})$, eccentric $(e=0.5)$ orbit. The somehow large distance of the star from the Sun $(d=62 \mathrm{pc})$ prevents the Hipparcos astrometric data to bring definitive constraints on the real mass of the planetary companion. Further details on the primary star, orbital elements and inferred planetary characteristics as well as the RV curve are given in Sivan et al. (this volume).

\subsection{HD 209458: spectroscopic transit}

The star HD 209458 became famous end of 1999 when the transit of a planet in front of the stellar disk was observed (Charbonneau et al. 2000, Henry et al. 2000), providing us for the first time with physical characteristics of an exoplanet (Mazeh et al. 2000). Observations with better facilities are now drastically improving these early results (see e.g. the impressive transit luminosity curve obtained with the HST by Brown et al., this volume). Here, we will concentrate on the transit observed spectroscopically with ELODIE in November 1999.

In the same way as spots, the shadow on the stellar disk of a transiting planet induces deformations of the spectral lines by hidding part of the light coming from the approaching or receding sides of the star. To see these deformations on individual lines requires very high-resolution and high signal-to-noise spectra whereas they can be easily observed on our cross-correlation functions which then are slightly red- or blue-shifted. This effect influences the measured RVs in the form of an anomaly of the orbital curve (Fig. 1, right) whose shape depends on the geometry of the transit and on stellar parameters. If the orbital and the apparent stellar equatorial planes are parallel, the lobes of the anomaly curve are symmetric, also the larger the stellar rotation, the larger the amplitude 
of the observed anomaly (Eggenberger 2000, master thesis; Queloz et al. 2000). The main results can be summarized as follow (Fig. 1, right): 1) the positive sign of the anomaly curve during the ingress indicates that the stellar rotation is in the same direction as the planet motion; 2) an angle $\Delta L \simeq 3.9^{\circ}$, but with large uncertainty, is estimated between the rotation and orbital axes; 3 ) the method provides an independent estimate of the stellar rotation: $v \sin i=3.75 \mathrm{kms}^{-1}$.

\section{The CORALIE planet-search programme: new results}

Since the summer of 1998, a large high-precision RV survey is being carried out by our Geneva group with the CORALIE echelle spectrograph on the 1.2-m Euler Swiss telescope at ESO-La Silla. CORALIE is the little sister of ELODIE with improved resolution $(R \simeq 50000)$ and smaller CCD pixels which allow to reach, with the same cross-correlation technique, a precision of $5-7 \mathrm{~ms}^{-1}$.

The CORALIE planet-search sample consists of more than 1650 F8 to M0 solar-type stars selected according to distance in order to obtain a statistically well-defined volume-limited set of dwarfs of the solar neighbourhood (Udry et al. 2000). In addition to the planet search, this sample will allow to collect information on spectroscopic binaries and give us a synthetic view on companions to solar-type stars from $q=m_{2} / m_{1}=1$ down to $q \leq 0.001$.

The CORALIE programme is very fruitful. In about 2 years, it has already revealed 19 low-mass companions with $m_{2} \sin i \leq 16 \mathrm{M}_{\mathrm{Jup}}$, among which 16 have minimum masses well in the planet domain $\left(m_{2} \sin i<5 \mathrm{M}_{\mathrm{Jup}}\right)$. As measurements are pilling up, the detection frequency is rising: 15 among the 19 candidates were announced between January and August 2000 (Naef et al. 2000; ESO 2000; this conference). From the observed frequency of giant planets, we expect about 100 planets out of the CORALIE sample.

In addition to the new ELODIE candidate, five new planets and one very low-mass brown dwarf detected with CORALIE are announced. Two (HD $83443 \mathrm{c}$ and HD $168443 \mathrm{c}$ ) of them are part of multi-component systems and they are described below, and four are additional planetary candidates orbit the stars HD 6434, HD 19994, HD 92788 (also presented by Butler et al. 2000, this volume) and HD 121504. The corresponding main stellar and orbital characteristics as well as inferred planetary properties and RV curves are given in Queloz et al. (this volume). In summary, 2 candidates have "intermediate" periods and low minimum masses (HD 6434: $m_{2} \sin i=0.48 \mathrm{M}_{\mathrm{Jup}}, P=22.1 \mathrm{~d}$; HD 121504: $m_{2} \sin i=0.89 \mathrm{M}_{\mathrm{Jup}}, P=64.6 \mathrm{~d}$ ), and two heavier planets are on long-period orbits (HD 19994: $P=454 \mathrm{~d}, \quad m_{2} \sin i=2.0 \mathrm{M}_{\mathrm{Jup}}: \quad$ HD $92788, \quad P=340 \mathrm{~d}$, $\left.m_{2} \sin i=3.8 \mathrm{M}_{\mathrm{Jup}}\right)$. The new candidate characteristics are very similar to properties of previously detected planets. In particular, the orbits are eccentric when the period is not too short.

\subsection{HD 83443: a 2-planet system with Saturn and sub-Saturn masses}

The most interesting new CORALIE candidate is the 2-planet system orbiting HD 83443. The short-period planet was already announced a few months ago in another set of 8 CORALIE new discoveries (ESO 2000). The period $P=2.985$ days is very close to 3 sidereal days. The planet has the shortest pe- 

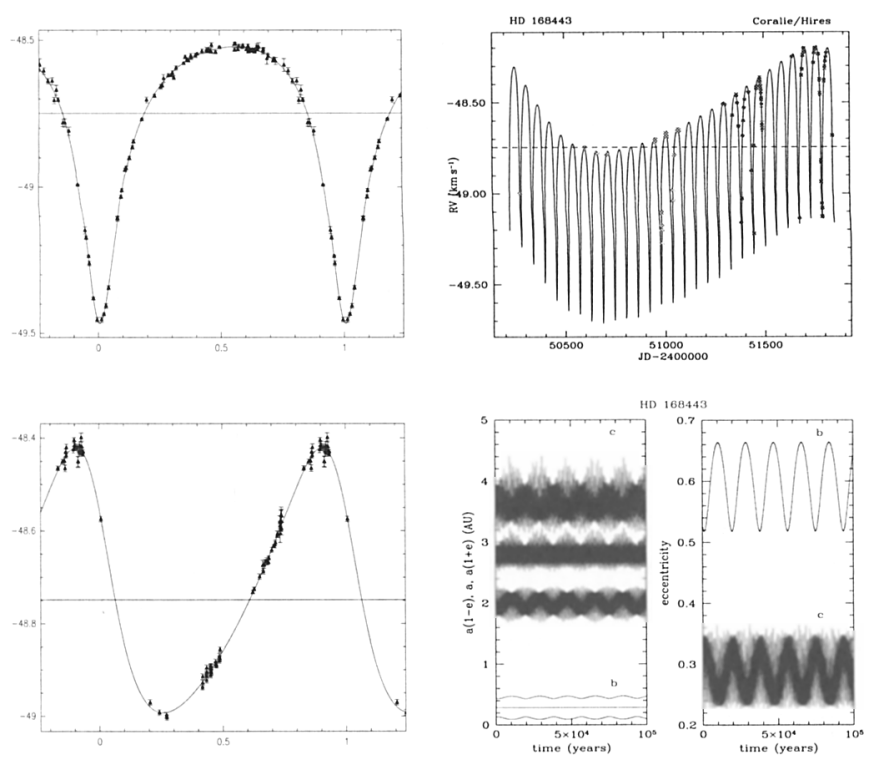

Figure 2. Simultaneously-derived orbital solutions for the HD 168443 system. The short (top left, b) and long (bottom left, c) periods are shown individually. The combined orbit is displayed as well (top right). Lower right. Stability analysis: temporal evolution over $10^{5}$ years of the eccentricity and separation parameters for both (b and c) orbits

riod and the smallest separation to the parent star among the known extra-solar planets. Early on, an abnormal behavour was detected in the residuals of the short-period Keplerian solution showing a periodicity of $P=29.83$ days (almost exactly 10 times the inner period). Very low masses are inferred for the planets, respectively close to and of half the mass of Saturn.

Looking at such a system with 2 planets on so close orbits immediately rises concerns about the system dynamical stability. A preliminary analysis clearly confirms the system stability. Nevertheless dynamical perturbations between the planets are far from being negligible and in particular may explain the noncircularity of the inner orbit despite of its very short period. Further orbital details and comments are given in Mayor et al. (this volume).

\subsection{HD 168443, a 3rd system: a planet + a low-mass brown dwarf}

Another intriguing system, composed of a heavy giant planet of $\sim 7.2 \mathrm{M}_{\mathrm{Jup}} / \sin i$ on a 58-d period orbit + a still heavier companion $\left(m_{2}=15.1 \mathrm{M}_{\mathrm{Jup}} / \sin i\right)$ further out, orbits HD 168443. The inner planet was announced by Marcy et al. (1999b) but with a much smaller mass $\left(m_{2}=4 \mathrm{M}_{\mathrm{Jup}} / \sin i\right)$. Their RVs combined with our CORALIE observations allow us to simultaneously derive Keplerian solutions for the 2 companions. Stellar and orbital parameters, inferred companion characteristics and RV curves are given in Table 1 and Fig. 2. The outer period is slightly 
larger than 1660 days. The period has not been completely covered yet but the orbital solution is already very robust, the minimum and maximum phases of the curve being covered. As for HD 83443 a preliminary stability study has been performed for the system by W. Benz (Fig. 2, lower right). Strong dynamical interactions between the components on various time scales (some related to the orbital periods) are clearly visible on the eccentricity and separation temporal variations. In particular, the large eccentricity value measured for the inner orbit $(e=0.52)$ is very probably due to the gravitational perturbation of the heavier outer companion. The system remains however stable.

The properties of HD 168443, if not due to an unlikely orbital plane inclination for both objects, set very interesting questions on the possible formation of such systems. The massive inner planet and the low-mass brown-dwarf companion are located well within the ice limit of the young protoplanetary disk. According to the migration scenario both objects had thus to move simultaneously towards the central region of the system. Simulations by Kley (2000) explore this possibility and show that in such a case, the outer object accreates disk material more efficiently. This rises the possibility of creating in the disk "super planets" with masses larger than $15 \mathrm{M}_{\mathrm{Jup}}$. On the other hand, if not in the disk the more massive outer companion is close enough to have truncated the disk within the ice limit thus preventing the giant planet to form.

\section{Orbital element distributions: fossil traces of planet formation}

\subsection{The mass function of substellar companions}

The observed gap in the mass distribution between giant planets and stellar secondaries (Fig. 3) strongly suggests the existence of two distinct companion populations for solar-type stars. The huge planetary peak is not the tail of the binary distribution. Despite the huge observational bias against the detection of small mass companions we observe an increasing number of low-mass planets. The planetary mass function even increases towards the lower masses. In the same time, the easier-detected brown dwarfs are rare. This is further emphasized when orbital plane inclinations can be derived e.g. with Hipparcos astrometric data (Halbwachs et al. 2000, Zucker \& Mazeh 2000), transit observations or synchronisation considerations.

The sharp drop of the mass function observed around $8 \mathrm{M}_{\mathrm{Jup}}$ strongly suggests a maximum mass for giant planets close to $10 \mathrm{M}_{\mathrm{Jup}}$. In particular, the shape of the mass function does not suggest a relation between the D-burning limit and the maximum mass for giant planets ${ }^{1}$.

\subsection{Short-period and eccentricity distributions}

Due to the still strong bias affecting the detection of long-period planets, a significant comparison between planetary candidates and spectroscopic binaries should be restricted to short-period systems. Figure 4 (left) focuses on $P \leq$

\footnotetext{
${ }^{1}$ The discovery of "free-floating brown dwarfs" in $\sigma$-Orionis with masses probably below $10 \mathrm{M}_{\mathrm{Jup}}$ (Zapatero Osorio et al. 2000) further refutes the D-burning limit as a good indicator of the brown dwarf - planet transition
} 

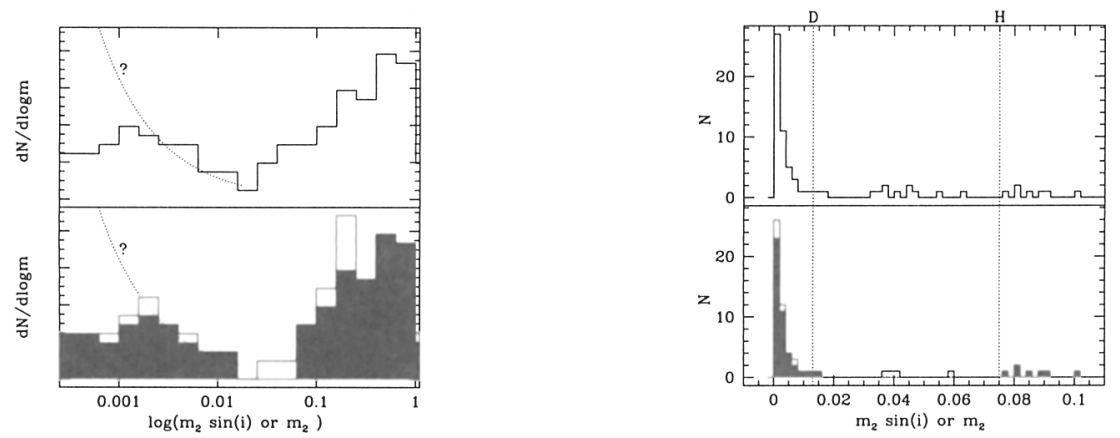

Figure 3. Mass function of companions to solar-type stars, including our latest detections, in log (left) and linear (right) scales. The dotted vertical lines indicate the $\mathrm{H}-$ and D-burning limits. Top: $m_{2} \sin i$. Bottom: composite histograms of $m_{2}$ (open part) and $m_{2} \sin i$ (if $\sin i$ not known; colored part)

10 days, comparing the corresponding cumulative distributions. The planetary period distribution is steeply rising for decreasing periods down to about 3 days reaching a clear cut-off. Several reasons may be invoked to set this limit like e.g. central cavity of the accretion disk, tidal interaction, Roche lobe overflow or evaporation.

Concerning orbital eccentricities, the comparison between spectroscopic binaries and giant planets is remarkable (an $(e, \log P)$ comparison diagram is given in Mayor \& Udry 2000). No obvious difference appears except in particular domains of periods. For periods larger than about 50 days, we cannot see any significant distinctions between both populations (Fig. 4, right) even if the origin of the eccentricity is supposed to be different (e.g. Mayor \& Udry 2000 for a further discussion). Nevertheless, a clear difference may be noticed between the two populations for periods between 10 and 50 days (Fig. 4, right). In this range of periods, outside the circularization domain, planetary systems have smaller eccentricities than stellar binaries indicating different formations or evolutions. Quasi-circular long-period binary orbits are very rare. Despite the observational bias a similar situation already exists for extra-solar giant planets. No solar-system analog has been discovered yet.
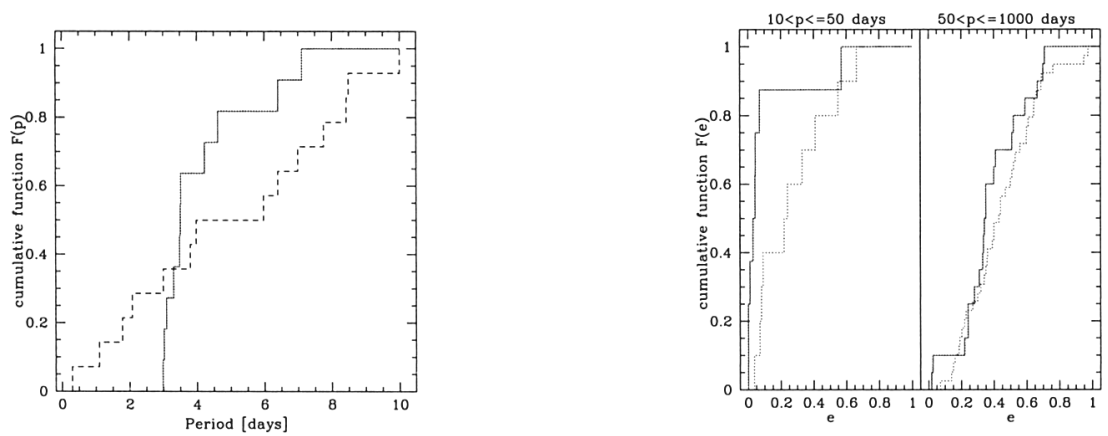

Figure 4. Cumulative distributions for planetary (solid line) and stellar companions (dashed line) to solar-type stars of: periods smaller than 10 days (left) and eccentricities for two domains of periods (right) 
In conclusion, the differences observed between planetary systems and binaries in the metallicity content of the primaries (see e.g. Santos, Isrealian \& Mayor, this volume; Santos et al. 2000), in the secondary mass functions and in the period and eccentricity distributions argue for the two populations being formed by distinct processes. More observations are however still needed to bring clear constraints on the possible formation and evolution scenarios.

\section{References}

Baranne A., Queloz D., Mayor M. et al. 1996, A\&AS, 119, 373

Charbonneau D., Brown T., Latham D., \& Mayor M. 2000, ApJ, 529, L45

Delfosse X., Forveille T., Mayor M. et al. 1998, A\&A, 338, L67

ESO 2000, http://www.eso.org/outreach/press-rel/pr-2000/pr-13-00.html

Halbwachs J.-L., Arenou F., Mayor M., Udry S., \& Queloz D. 2000, A\&A, 355, 581

Henry G., Marcy G., Butler P., \& Vogt S. 2000, ApJ, 529, L41

Kley W. 2000, MNRAS, 313, L47

Mazeh T., Naef D., Torres G. et al. 2000, ApJ, 532, L55

Marcy G., Butler P., Vogt S., Fischer D., \& Lissauer J. 1999a, ApJ, 505, L147

Marcy G., Butler P., Vogt S., Fischer D., \& Liu M. 1999b, ApJ, 520, 239

Mayor M., \& Queloz D. 1995, Nature, 378, 355

Mayor M., \& Udry S. 2000, in "Disks, planetesimals and planets", Tenerife, ASP Conf. Ser., in press

Naef D., Mayor M., Pepe F. et al. 2000b, A\&A, in press

Queloz D., Eggenberger A., Mayor M. et al. 2000, A\&A, 359, L13

Santos N.C., Israelian G., \& Mayor M. 2000, A\&A, in press

Udry S., Mayor M., \& Naef D., et al. 2000, A\&A, 356, 590

Zapatero Osorio M.-R., Béjar V., Martin E. et al. 2000, Science, 290, 103

Zucker S., \& Mazeh T. 2000, ApJ, 531, L67 